\title{
Condensed tannin: a major anti-nutritional constituent of faba bean (Vicia faba L.)
}

\begin{abstract}
Nutritionally faba bean (Vicia faba L.) is one of the important legume crops cultivated for both food and feed purposes. However, in some parts of the world it has not gained status of commercial legume crop and continues to be grown in marginal agricultural fields with almost no agricultural inputs. Despite of having nutritional as well as medicinal properties, it is not being cultivated in large scale because of one or other anti-nutritional constituents present in its edible parts. Condensed tannins are one of the major anti-nutritional factors found in edible parts of this crop which confers noticeable astringency and inhibition in digestion process. Molecular breeding and genetic engineering approaches may provide successful way to improve this crop to reduce the content of condensed tannin in the seed coat. Germplasm characterization for identification of best parents and identification of crucial genes/QTLs are some of the initial steps for improvement of this crop. Genomic information available in model and other legume crops will also serve as important resources for taking suitable approach.
\end{abstract}

Keywords: Proanthocyanidins, anti-nutritional factors, food legumes, phenolics, flavan-3-ols
Volume 2 Issue 2 - 2018

\author{
Subodh Kumar Sinha, Amresh Kumar \\ ICAR-National Research Centre on Plant Biotechnology, Pusa \\ Campus, India
}

\author{
Correspondence: Subodh Kumar Sinha, ICAR-National \\ Research Centre on Plant Biotechnology, Pusa Campus, India, Tel \\ +91-I I-2584 I 787, Fax +9 |- I I-25843984,
}

Email subsinha@gmail.com

Received: January 29, 2018| Published: April II, 2018

\section{Introduction}

Faba bean (Vicia faba L.) is one of the oldest crops grown by men to supply high-protein seeds for human and animal nutrition which is known by variety of common names such field bean, broad bean, horse bean, tick bean, windsor bean, hava feve, baakla shim. This legume crop is an important food legume in China, Egypt, Italy, Brazil and Ethiopia, whereas in some of the countries like India it is one of the underutilized pulse crop which is mainly cultivated in few states of India on a limited scale and sold in limited quantities to local markets and thus it has never achieved a status of a commercial pulse crop. Faba bean as a plant food for human consumption provides a balanced diet of lysine-rich protein, carbohydrates, fibre, and good source of iron, magnesium, potassium, zinc, copper, selenium, and many vitamins. Furthermore, faba bean has been attributed with certain medicinal values as it serves as source of levodopa, a natural precursor of dopamine that has potential use in the treatment of Parkinson's disease. Levodopa was identified in the seedlings, pods and beans of the faba bean (Vicia faba) by Guggenheim. ${ }^{1}$ When taken orally, levodopa is absorbed into the blood stream and carried to the brain where it is converted into dopamine. On contrary, faba bean is also known for its high content of phenolic compounds for instance condensed tannins which serve to confer better suitability of the crop in undesirable external environments. However, much attention is now being placed on the antioxidant capacity of the flavonoids and the phenolics of this crop. ${ }^{2}$ However, a drawback to the more widespread use of the broad bean stems from its toxicity because it contains some antinutritional factors (ANFs) viz. condensed tannins, trypsin inhibitor activity (TIA), which are located in the testa; lectins, pyrimidine glucosides (vicin and convicine) which accumulate in the cotyledons; all of them negatively affect their digestibility and other nutritive quality. Condensed tannins (CTs) are one the most important ANFs located in the testa, which negatively affect their digestibility and other nutritive value. The flavan-3-ols are the monomers of condensed tannins (CTs, also known as proanthocyanidins PAs), which are widely found in seed coats, leaves, flowers, stems and other tissues throughout the plant kingdom.

Chemically tannins have been referred for those phenolic compounds whose molecular weight $(>500)$ is sufficiently high to form a reasonably strong complexes with proteins and other polymers under suitable condition of concentration and $\mathrm{pH}$. The dry or puckery sensation of astringency, which happens during the ripening of many edible fruits, undoubtedly arises from the cross-linking of the proteins and glycoproteins in the mouth by tannins with a correspondingly reduced lubricant action. These cross-linking is probably mainly achieved by the formation of hydrogen bonds or other equivalent weaker interactions. The molecular size, the overall structure, and the number and orientation of phenolic hydroxyl groups of tannins are largely responsible for the actual bond formation. ${ }^{3}$ It has been observed that neither low molecular weight phenolic compounds nor highly polymerized tannins show noticeable astringency, rather maximum astringency is most probably shown by tannins of intermediate size. Tannins are classified into two groups: condensed tannins which are polymers of flavonols and yield anthocyanidins when heated in acid solution, and hydrolysable tannins which produce gallic acid as a degradation product. The latter are easily eliminated with the action of the gastric juices and do not represent a problem in animal feeding. Of these two types, condensed tannins are considered as the major ANFs. The antinutritional and toxic effects of tannins have been categorized as: depression of food intake, inhibition of digestive enzymes, formation of less digestible tannin-dietary protein complexes, increased excretion of proteins, digestive tract malfunctions and toxicity of absorbed tannin or its metabolites. ${ }^{4}$ The adverse effect of tannins is because of their astringent taste, binding ability with digestive enzymes or exogenous protein as well as precipitation of salivary proteins by solubilized tannins. ${ }^{4}$

CTs share the same upstream biosynthestic pathway as anthocyanin. The building blocks or starter units of most PAs are 
the flavan-3-ols catechin and epicatechin, whose last common biosynthetic intermediate is leucoanthocyanidin (an intermediate in anthocyanin biosynthesis also referred to as flavan 3,4-diol), which results from the reduction of flavonols by dihydroflavonol 4-reductase (DFR). ${ }^{5}$ Subsequently, catechin is derived from the reduction of leucoanthocyanidin through the activity of leucoanthocyanidin reductase (LAR). Epicatechin formation occurs via anthocyanidin synthesis and reduction, two steps catalyzed by anthocyanidin synthase (ANS) and anthocyanidin reductase (ANR), encoded by leucoanthocyanidin dioxygenase (LDOX)/ANS and BANYULS (BAN)/ANR genes, respectively. Anthocyanidins are consequently essential for PA biosynthesis in species that accumulate only epicatechin-based PAs. Chemical characterization of the reaction products of ANR identified two isomers of flavan-3-ol, namely $2 \mathrm{R}$, 3R-2, 3-cis-and 2S, 3R-2, 3-trans. With cyanidin as substrate, these products are (-)-epicatechin and (-)-catechin, respectively.The2R, 3R-2, 3-cis-flavan-3-ol, which was the major reaction product, is the common building block of CTs in many plants including Arabidopsis and alfalfa (Medicago sativa).

\section{Way forward for improvement}

Characterization of the genetic variation in the available germplasms is important for further crop improvement which facilitates the transfer of useful genes among cultivated species and to improve yield parameters to impart resistance to biotic and abiotic stresses. ${ }^{6}$ Our work demonstrated that different faba bean cultivars have different level of extractable phenol and proanthocynidin ${ }^{7}$ in seed extract (Figure 1A). The whole tissue or histo-chemical localization of CTs may serve the purpose to determine its content in different germplasms for selection of better parent for conventional and molecular breeding to address this nutritional problem of this pulse crop. We used CT specific reagent known as DMACA (p-dimethylaminocinamaldehyde) reagent in concentration of $2 \% \mathrm{w} / \mathrm{v}$ DMACA in $3 \mathrm{M} \mathrm{HCl}$ for different time period for staining CTs in seed coat of whole seed and its localization (Figure 1B) (Figure 1C). Plants exhibit both carbon and nitrogen based defense system depending on environmental and their growth conditions. ${ }^{8}$ Probably because of this reason the amount of PAs are regulated in various tissues to better adapt in different external conditions (Figure 1D). A considerable amount of PA may be removed simply by soaking in water. Our results show 30 hrs of soaking resulted around $45 \%$ of decrease in PA content in seed and there after no considerable decrease was observed (Figure 1E). As far as genetic engineering approaches are concerned, down regulation of ANR gene in seed by RNA interference techniques may facilitate silencing of its expression and hence modulate amount of condensed tannins in the end product of faba bean to improve its nutritional quality.

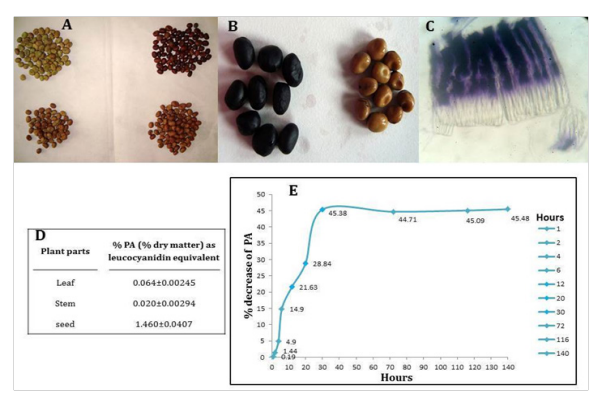

Figure 1 A: Different faba bean germplasms showing diverse seed coat color at maturity; B: whole seed; C: seed coat stained with DMACA; D: \% PA content in different parts of faba bean; E: \% decrease in PA content in water leachate.

\section{Conclusion}

Considering the nutritional aspects and minimal requirement of agricultural inputs of faba bean, this pulse crop has potential to be used as commercial pulse crop provided the content of antinutritional constituents specifically in economically important plant parts is reduced. The current genomic and marker-assisted breeding technologies and basic understanding about secondary metabolic pathway will serve the potential ways to improve the nutritional quality of this pulse crop.

\section{Acknowledgements}

SKS acknowledges the funding received under Non-Plan Research Grant (252/DR Dated 18/06/2010) of Dr. Rajendra Prasad Central Agricultural University, Pusa, India (erstwhile Rajendra Agricultural University, Pusa).

\section{Conflict of interest}

Authors declare no conflict of interest.

\section{References}

1. Guggenheim M. Dioxyphenylalanain, A new amino acid from Vicia faba. Z Physiol Chem. 1913;88:276-284.

2. Sinha SK, Kumar M, Kumar A, et al. Antioxidant activities of different tissue extract of Faba bean (Vicia faba L.) containing phenolic compounds. Legume Res. 2013;36(6):496-504.

3. Goldstein JL, Swain T. Changes in Tannins in Ripening Fruits. Phytochemistry. 1963;2(4):371-383.

4. Jansman AJM, Longstaff M Nutritional effects of tannins and vicine/ convicine in legume seeds. In Recent Advances of Research in Antinutritional Factors in Legume Seeds. Proceedings of the 2nd International Workshop on 'Antinutritional Factors (ANFs) in Legume Seeds', Wageningen, The Netherlands, EAAP Publication No. 70, AFB Van der Poel, J Huisman, HS Saini, editors. Wageningen, the Netherlands: Wageningen Press; 1993:301-316.

5. Dixon RA, Xie DY, Sharma SB. Proanthocyanidins $-\mathrm{a}$ final frontier in flavonoid research? New Phytol. 2005;165(1):9-28.

6. Pradhan A, Shahi VK, Sinha SK. Evaluation of Genetic Diversity in Faba bean (Vicia faba L.) Genotypes using Seed Protein and Isozymes Electrophoresis. Natl Acad Sci Lett. 2014;37(4):303-309.

7. Kumar A, Nidhi, Prasad N, et al. Nutritional and antinutritional attributes of faba bean (Vicia faba L.) germplasms growing in Bihar, India. Physiol Mol Biol Plants. 2015;21(1):159-162.

8. Bryant JP, Chapin FS, Klein DR. Carbon/Nutrient balance of boreal plants in relation to vertebrate herbivory. Oikos. 1983; 40(3):357-368. 